\title{
Dynamics of longitudinal phase-space modulations in an rf compressor for electron beams
}

\author{
M. Venturini* \\ LBNL, Berkeley, California 94720-8211, USA \\ M. Migliorati ${ }^{\dagger}$ \\ SAPIENZA University of Rome, Rome, Italy \\ C. Ronsivalle \\ ENEA, Centro Ricerche Frascati, Rome, Italy
}

M. Ferrario and C. Vaccarezza

INFN-LNF, Frascati, Rome, Italy

(Received 9 May 2010; published 5 August 2010)

\begin{abstract}
Free-electron lasers operating in the UV or x-ray radiation spectrum require peak beam currents that are generally higher than those obtainable by present electron sources, thus making bunch compression necessary. Compression, however, may heighten the effects of collective forces and degrade the beam quality. In this paper we provide a framework for investigating some of these effects in rf compressors by focusing on the longitudinal dynamics of small-amplitude density perturbations, which have the potential to cause the disruptive appearance of the so-called microbunching instability. We develop a linear theory valid for low-to-moderate compression factors under the assumption of a 1D impedance model of longitudinal space charge and provide validation against macroparticle simulations.
\end{abstract}

DOI: 10.1103/PhysRevSTAB.13.080703

PACS numbers: 41.75.Fr, 41.85.Ew, 29.27.Bd

\section{INTRODUCTION}

Electron beam applications, and in particular those for $\mathrm{x}$ ray free-electron lasers (FELs), often require high peak currents that can only be obtained through compression downstream of the beam source. The conventional technique for compression exploits the energy dependence of a particle orbit path length through magnetic chicanes. While effective, magnetic compression can spoil the beam transverse emittance because of coherent radiation emission in the bends. In contrast, compression by velocity bunching (or rf compression, RFC) taking place as the beam travels through an rf structure is immune to radiation effects, and represents a potentially attractive alternative [1]. Recent measurements [2] have demonstrated that there exist useful ranges of beam parameters for which rf compression can be achieved without compromising emittance compensation, suggesting that it could be a viable option for generation of high-brightness beams. Indeed, compression schemes relying at least in part on rf compression are currently under active investigation in the design of next generation light sources [3].

An important, but still unexplored, aspect of rf compression concerns the preservation of longitudinal phase-space quality. It is known that beam compression through chicanes can trigger the so-called microbunching instability causing small density perturbations to evolve into large and

\footnotetext{
*MVenturini@lbl.gov

${ }^{\dagger}$ Also at INFN-LNF, Frascati, Rome, Italy.
}

undesirable fluctuations in phase space [4-7]. In principle, microbunching can also affect rf compression as the two basic ingredients for the instability, collective forces and non-negligible dependence of a particle time of flight on energy, are both present. The low beam energy at which rf compression takes place causes space charge to be fairly strong, while at the same time generating significant dispersion even in the absence of bending magnets (indeed it is this very feature that makes rf compression possible).

In this paper we present a linear theory for the evolution of small-amplitude density perturbations in the beam longitudinal phase space through an rf compressor. The method we apply is similar to that developed for the study of the microbunching instability in magnetic compressors $[5,6]$. A number of approximations are introduced in order to keep the problem in manageable form. First, the singleparticle motion of an electron in the rf structure is treated in the linear approximation, which limits the applicability of the model to low or moderate compression factors (say up to a factor 3 or 4). A second approximation results from adopting a 1D model for the space charge impedance describing collective effects, which at smaller wavelengths and low energy is known to be problematic [8,9]. Finally a coasting beam approximation is assumed, which limits the applicability to particle dynamics in the longitudinal core of the physical electron bunches.

To validate the analytical model and provide a check on the simplifying approximations, we also present a comparison against macroparticle simulations. We find that a reasonable agreement with the theory can be obtained over 
a wide spectrum of perturbation wavelengths provided that certain empirical modifications in the parameters for the longitudinal space charge impedance be introduced. We ascribe the need for this adjustment to the limitations of the adopted 1D model for space charge.

The paper is organized as follows. After deriving in Sec. II the transfer map for single-particle motion through an rf structure in the linear approximation, in Sec. III we present the small-amplitude perturbation theory. The main result is the derivation of an integral equation obeyed by the beam bunching function expressing the evolving amplitude of an initial sinusoidal density modulation through the compressor. We follow with a discussion of the numerical model used for the macroparticle simulations and a comparison of simulations against theory (Sec. IV).

\section{SINGLE-PARTICLE DYNAMICS THROUGH AN RF COMPRESSOR}

We assume that compression takes place in a traveling wave rf structure. The longitudinal motion of an electron in such a structure is described by the Hamiltonian $H=$ $\sqrt{m^{2} c^{4}+p_{s}^{2} c^{2}}-e \phi(s, t)$, where $-e$ is the electron charge, $s$ the longitudinal coordinate, and $\phi=\left(E_{0} / k_{\mathrm{rf}}\right) \times$ $\cos \left(k_{\mathrm{rf}} s-\omega_{\mathrm{rf}} t+\psi_{0}\right)$ the electric potential, yielding the longitudinal electric field $E_{s}=-\partial \phi / \partial s=E_{0} \sin \left(k_{\mathrm{rf}} s-\right.$ $\left.\omega_{\mathrm{rf}} t+\psi_{0}\right)$. For simplicity, in the following we assume a phase velocity $\omega_{\mathrm{rf}} / k_{\mathrm{rf}}=c$ for the traveling wave. The canonical equations are readily written: $d s / d t=$ $p_{s} c^{2} / \sqrt{m^{2} c^{4}+p_{s}^{2} c^{2}}$ and $d p_{s} / d t=-e E_{0} \sin \psi$, where we have introduced the phase $\psi=k_{\mathrm{rf}} s-\omega_{\mathrm{rf}} t+\psi_{0}$.

We then change the dynamical coordinates from $s$ and $p_{s}$ to $t$ and the relativistic factor $\gamma=\sqrt{m^{2} c^{4}+p_{s}^{2} c^{2}} / m c^{2}$, while turning $s$ into the independent variable. The resulting equations for $t(s)$ and $\gamma(s)$ read

$$
\frac{d t}{d s}=\frac{\gamma}{c \sqrt{\gamma^{2}-1}}
$$

$$
\frac{d \gamma}{d s}=-\alpha k_{\mathrm{rf}} \sin \psi
$$

where we have introduced the parameter $\alpha=$ $e E_{0} /\left(m c^{2} k_{\mathrm{rf}}\right)$.

The orbit for the reference particle is a particular solution of (1) and (2), which we denote as $\left(t_{r}, \gamma_{r}\right)$. The orbit of any other particle can be described in terms of the deviation variables $\Delta t=t-t_{r}$ and $\Delta \gamma=\gamma-\gamma_{r}$, where $(t, \gamma)$ are also solutions of (1) and (2). It is convenient to introduce the space separation $\Delta z=z-z_{r}$ between an electron and the reference particle, in place of $\Delta t$. We have $\Delta z=$ $-c \beta(s) \Delta t$, where $c \beta(s)$ is the beam velocity. The negative sign results from the convention that for a particle in the head of the bunch $\Delta z>0$. In the following for the purpose of determining $\Delta z$ from $\Delta t$, we will assume that the beam is sufficiently relativistic so that $\beta \simeq 1$, yielding $\Delta z \simeq$ $-c \Delta t$.

The equations of motion for the deviation variables $\Delta z$ and $\Delta \gamma$ read

$$
\begin{gathered}
\frac{d \Delta z}{d s}=\frac{\gamma_{r}}{\sqrt{\gamma_{r}^{2}-1}}-\frac{\gamma_{r}+\Delta \gamma}{\sqrt{\left(\gamma_{r}+\Delta \gamma\right)^{2}-1}}, \\
\begin{aligned}
\frac{d \Delta \gamma}{d s}= & \alpha k_{\mathrm{rf}}\left[\sin \left(k_{\mathrm{rf}} s-\omega_{\mathrm{rf}} t_{r}+\psi_{0}\right)\right. \\
& \left.-\sin \left(k_{\mathrm{rf}} s-\omega_{\mathrm{rf}} t_{r}+k_{\mathrm{rf}} \Delta z+\psi_{0}\right)\right] .
\end{aligned}
\end{gathered}
$$

The first-order solution of the above linear system can be expressed in terms of the transfer matrix $\boldsymbol{M}: \boldsymbol{x}(s)=\boldsymbol{M} \boldsymbol{x}_{0}$, where $\boldsymbol{x}(s)=[\Delta z(s), \Delta \gamma(s)]$ and $\boldsymbol{x}_{0}=\boldsymbol{x}\left(s_{0}\right)$, with matrix $M$ obeying

$$
\frac{d \boldsymbol{M}}{d s}=\boldsymbol{A} \boldsymbol{M}
$$

with initial condition $\boldsymbol{M}\left(s_{0}\right)=\mathbf{1}$ and matrix $\boldsymbol{A}$ defined by

$$
\boldsymbol{A}(s)=\left(\begin{array}{cc}
0 & {\left[\gamma_{r}^{2}(s)-1\right]^{-3 / 2}} \\
-\alpha k_{\mathrm{rf}}^{2} \cos \left(k_{\mathrm{rf}} s-\omega_{\mathrm{rf}} t_{r}(s)+\psi_{0}\right) & 0
\end{array}\right)
$$

Incidentally, the form of the matrix (6) allows us to infer immediately that the transformation $\boldsymbol{M}$ is symplectic.

Examples of solutions of (1), (2), and (5) for the reference orbit and entries of the transfer matrix $\boldsymbol{M}$ are shown in Figs. 1 and 2, respectively. Here and in the examples to follow we make reference to a $3 \mathrm{~m}$ long, S-band, SLACtype, traveling wave section currently in use for the SPARC experiments [10]. The rf structure frequency is $2856 \mathrm{MHz}$ (corresponding to $\lambda_{\mathrm{rf}}=10.5 \mathrm{~cm}$ or $k_{\mathrm{rf}}=59.8 \mathrm{~m}^{-1}$ ). We assume a $E_{0}=25 \mathrm{MeV} / \mathrm{m}$ peak accelerating gradient (yielding $\alpha=0.82$ ). The rf structure is preceded by a $0.6 \mathrm{~m}$ long drift and the electron beam injected with $5.6 \mathrm{MeV}$ kinetic energy $\left(\gamma_{r 0} \simeq 12\right)$. The reference orbit is best represented in terms of the variable $\zeta_{r}$, defined as $\zeta_{r}=$ $s-c t_{r}$ (Fig. 2, right). This quantity expresses the relative longitudinal distance between the reference electron and an imaginary particle traveling at the speed of light. The initial conditions were specified so that as the reference particle enters the structure $(s=0.6 \mathrm{~m})$ we have $\zeta_{r}=0$, i.e., the rf phase is $\psi=\psi_{0}$. 

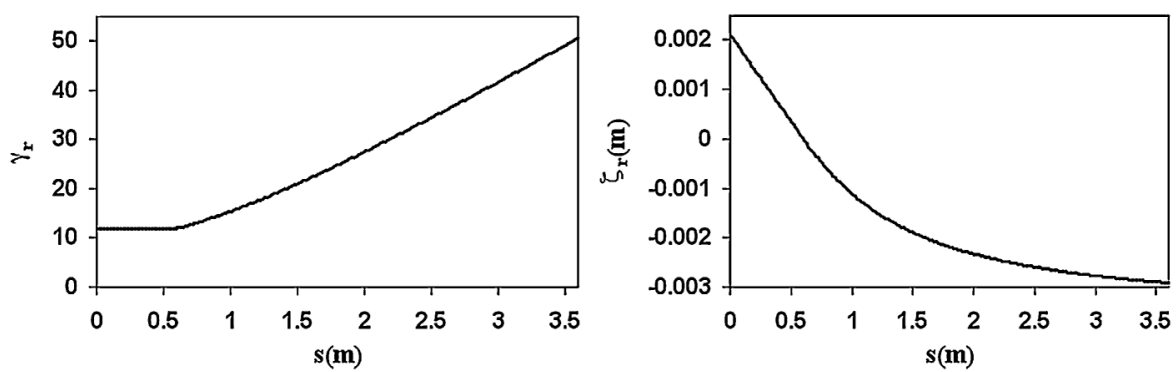

FIG. 1. Example of electron reference orbit through an rf compressor obtained by numerical solution of Eqs. (1) and (2). The left picture shows the evolution of the relativistic $\gamma$ factor, the right picture the relative longitudinal distance between the reference electron and an imaginary particle traveling at the speed of light. (The relevant parameters are $k_{\mathrm{rf}}=59.8 \mathrm{~m}^{-1}, \alpha=0.82, \psi_{0}=-8 \mathrm{deg}$, and $\left.\gamma_{r 0}=12.\right)$
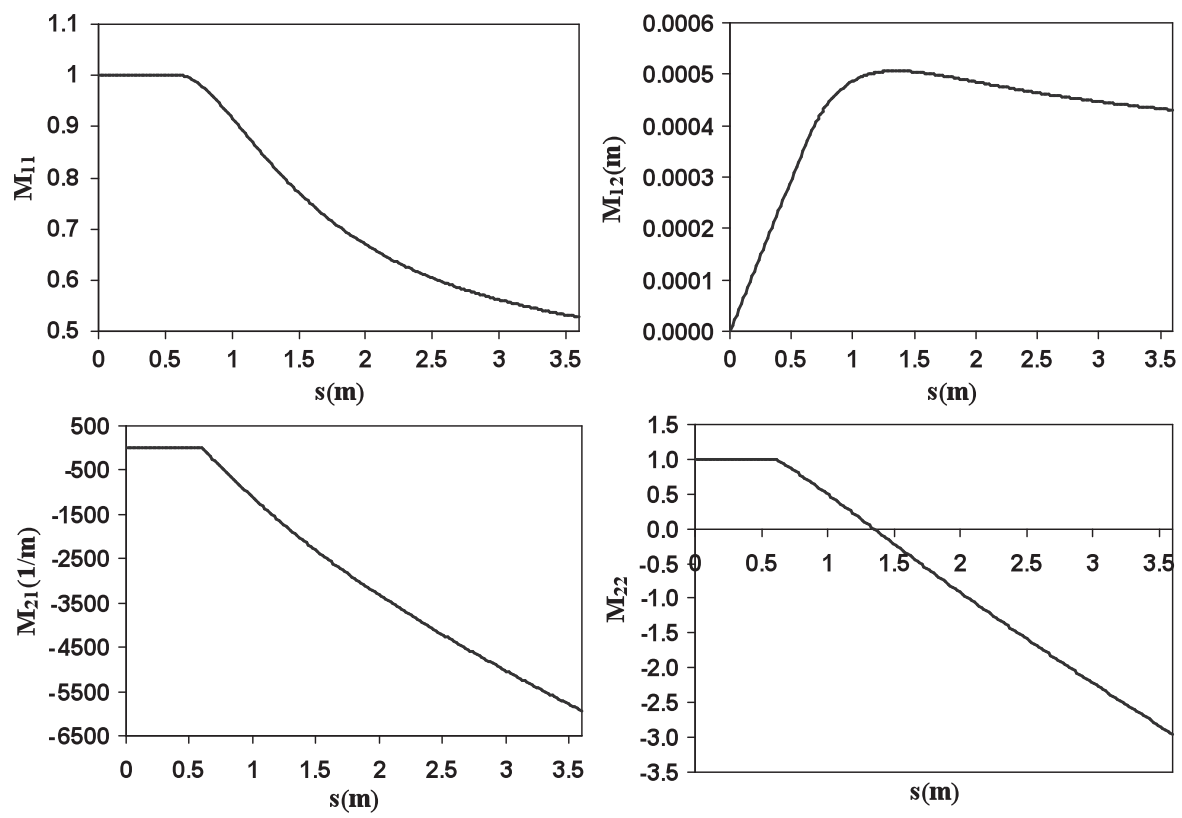

FIG. 2. Entries of the transfer matrix $\boldsymbol{M}$ for $k_{\mathrm{rf}}=59.8 \mathrm{~m}^{-1}, \alpha=0.82, \psi_{0}=-8 \mathrm{deg}$, and $\gamma_{r 0}=12$.

\section{LINEAR THEORY}

In the following we will refer to the dynamical variables in vector notation as $\boldsymbol{x}=(\Delta z, \Delta \gamma)$. Moreover, to simplify notation, we will use $z$ to denote $\Delta z$ and denote $\Delta \gamma$ with $p$, i.e. $\boldsymbol{x}=(z, p)$.

We assume that the beam distribution $f\left(x_{0} ; s_{0}\right)=$ $f_{0}\left(\boldsymbol{x}_{0} ; s_{0}\right)+f_{1}\left(\boldsymbol{x}_{0} ; s_{0}\right)$ at the entrance of the rf compressor $s=s_{0}$ consists of a zero-order smooth density, uniform in $z$ and Gaussian in $p$ with a chirp $h_{0}$,

$$
f_{0}\left(\boldsymbol{x}_{0} ; s_{0}\right)=\frac{1}{\sqrt{2 \pi} \sigma_{p}} e^{-\left(p_{0}-h_{0} z_{0}\right)^{2} / 2 \sigma_{p}^{2}},
$$

and a first-order perturbation $f_{1}\left(\boldsymbol{x}_{0} ; s_{0}\right)$. The normalization of the distribution function is chosen so that $n_{0} d z \int_{-\infty}^{\infty} d p_{0} f(x ; s)$ yields the number of particles in the interval $d z$, where $n_{0}$ is the beam line density.

Let us consider the evolution of the unperturbed beam density first. The beam density function $f_{0}\left(\boldsymbol{x}_{s} ; s\right)$ at $s$ is related to the beam density $f_{0}\left(\boldsymbol{x}_{0} ; s_{0}\right)$ at $s_{0}$ by $f\left(\boldsymbol{x}_{s}, s\right)=$ $f\left(\boldsymbol{M}^{-1} \boldsymbol{x}_{s} ; s_{0}\right)$. Here and in the following we use $\boldsymbol{M}, \boldsymbol{M}(s)$, or $\boldsymbol{M}\left(s_{0} \rightarrow s\right)$ interchangeably to denote the transfer matrix solution of (5) from $s=s_{0}$ to $s$ and use the notation $\boldsymbol{M}\left(s^{\prime} \rightarrow s\right)$ to represent the matrix advancing the linear solutions of (3) and (4) from $s^{\prime} \geq s_{0}$ to $s \geq s^{\prime}$, i.e. $\boldsymbol{M}\left(s^{\prime} \rightarrow\right.$ $s)=\boldsymbol{M}(s) \boldsymbol{M}^{-1}\left(s_{0} \rightarrow s^{\prime}\right)$.

The normalized charge density evolves from $\rho\left(z_{0} ; s_{0}\right)=$ $\int_{-\infty}^{\infty} d p_{0} f_{0}\left(\boldsymbol{x}_{0} ; s_{0}\right)=1$ at $s=s_{0}$ to

$$
\begin{aligned}
\rho\left(z_{s} ; s\right) & =\int_{-\infty}^{\infty} f_{0}\left(\boldsymbol{x}_{s}, s\right) d p_{s}=\frac{1}{\left|M_{11}(s)+h_{0} M_{12}(s)\right|} \\
& \equiv C(s) .
\end{aligned}
$$

The last equality in the above equation identifies the compression factor $C=C(s)$. In writing (8) we made use of the symplectic property of the matrix $\boldsymbol{M}$.

The effect of collective forces described by an impedance $Z(k ; s)$ (to be specified later) is to change the particle 
energy according to

$$
\frac{d p}{d s}=F\left(\tilde{\rho}, z_{s} ; s\right) \equiv-\frac{4 \pi I_{0}}{I_{A} Z_{0}} \int_{-\infty}^{\infty} d k e^{i k z_{s}} Z(k ; s) \tilde{\rho}(k ; s),
$$

where $I_{0}=e c n_{0}$ is the electron beam current, $I_{A}=$ $c e / r_{e} \simeq 17 \mathrm{kA}$ the Alfvén current, and $Z_{0}$ the vacuum impedance, with

$$
\tilde{\rho}(k ; s)=\frac{1}{2 \pi} \int_{-\infty}^{\infty} d z_{s} e^{-i k z_{s}} \int_{-\infty}^{\infty} d p f\left(\boldsymbol{x}_{s} ; s\right)
$$

being the Fourier transform (FT) of the charge density $\rho\left(z_{s}, s\right)$ at $s$.

The starting point of our analysis is the linearized Vlasov equation expressed in the integral form [6]:

$$
f_{1}\left(\boldsymbol{x}_{s} ; s\right)=f_{1}\left(\boldsymbol{x}_{0} ; s_{0}\right)-\int_{s_{0}}^{s} d s^{\prime} F\left(\tilde{\boldsymbol{\rho}}_{1} ; z_{s^{\prime}} ; s^{\prime}\right) \frac{\partial f_{0}\left(\boldsymbol{x}_{s^{\prime}} ; s^{\prime}\right)}{\partial p_{s^{\prime}}},
$$

with the collective force $F$ depending on the FT $\tilde{\rho}_{1}$ of the first-order density perturbation.

Starting from (11) our goal is to derive an equation for $\tilde{\rho}_{1}$. To this end it is convenient to think of both sides of (11) as functions of the dynamical variables at current position $s$ :

$$
\begin{aligned}
f_{1}\left(\boldsymbol{x}_{s} ; s\right)= & f_{1}\left[\boldsymbol{x}_{0}\left(\boldsymbol{x}_{s}\right) ; s_{0}\right]-\left.\int_{s_{0}}^{s} d s^{\prime} F\left(\tilde{\boldsymbol{\rho}}_{1} ; z_{s^{\prime}} ; s^{\prime}\right)\right|_{z_{s^{\prime}}=z_{s^{\prime}}\left(\boldsymbol{x}_{s}\right)} \\
& \times\left.\frac{\partial f_{0}\left(\boldsymbol{x}_{s^{\prime}} ; s^{\prime}\right)}{\partial p_{s^{\prime}}}\right|_{\boldsymbol{x}_{s^{\prime}}=\boldsymbol{x}_{s^{\prime}}\left(\boldsymbol{x}_{s}\right)},
\end{aligned}
$$

where $\boldsymbol{x}_{0}\left(\boldsymbol{x}_{s}\right)$ denotes the linear transformation $\boldsymbol{x}_{0}=\left[\boldsymbol{M}\left(s_{0} \rightarrow s\right)\right]^{-1} \boldsymbol{x}_{s}$ and similarly $\boldsymbol{x}_{s^{\prime}}\left(\boldsymbol{x}_{s}\right)=\left[\boldsymbol{M}\left(s^{\prime} \rightarrow\right.\right.$ $s)]^{-1} \boldsymbol{x}_{s}$.

The next step is to make a more definite assumption about the form of the initial perturbation. We assume an expression of the form

$$
f_{1}\left(\boldsymbol{x}_{0} ; s_{0}\right)=A e^{i k_{0} z_{0}} \frac{e^{-\left(p_{0}-h_{0} z_{0}\right)^{2} / 2 \sigma_{p}^{2}}}{\sqrt{2 \pi} \sigma_{p}},
$$

i.e. consisting of a sinusoidal perturbation to the charge density (while the $p$ density is the same as in the unperturbed distribution). As usual in this kind of calculation, the physically meaningful component is the real part of the complex quantity (13).

We are now ready to integrate both sides of Eq. (12) over the phase space after multiplying by $e^{-i k z_{s}}$. By definition, the FT of the left-hand side of Eq. (12) yields $\tilde{\rho}_{1}(k ; s)$. The FT of the first term on the right-hand side (RHS), which we denote with $I_{1}$, requires more work. First, we carry out the transformation of variables $\boldsymbol{x}_{s} \rightarrow \boldsymbol{x}_{0}$ and exploit symplecticity $d^{2} \boldsymbol{x}_{s}=d^{2} \boldsymbol{x}_{0}$ to write

$$
I_{1}=\frac{1}{2 \pi} \int_{-\infty}^{\infty} d z_{0} \int_{-\infty}^{\infty} d p_{0} f_{1}\left(\boldsymbol{x}_{0} ; s_{0}\right) e^{-i k z_{s}\left(\boldsymbol{x}_{0}\right)},
$$

where $z_{s}\left(\boldsymbol{x}_{0}\right)=\left[\boldsymbol{M} \boldsymbol{x}_{0}\right]_{1}=M_{11} z_{0}+M_{12} p_{0}$. We then insert expression (13) into (14),

$$
\begin{aligned}
I_{1}= & \frac{A}{2 \pi \sqrt{2 \pi} \sigma_{p}} \int_{-\infty}^{\infty} d p_{0} \int_{-\infty}^{\infty} d z_{0} e^{-\left(p_{0}-h z_{0}\right)^{2} / 2 \sigma_{p}^{2}} e^{i k_{0} z_{0}} \\
& \times e^{-i k\left(M_{11} z_{0}+M_{12} p_{0}\right)}
\end{aligned}
$$

and introduce the change of variable $t=p_{0}-h_{0} z_{0}$, yielding

$$
\begin{aligned}
I_{1} & =\frac{A}{2 \pi \sqrt{2 \pi} \sigma_{p}} \int_{-\infty}^{\infty} d z_{0} e^{i z_{0}\left(k_{0}-k / C\right)} \int_{-\infty}^{\infty} d t e^{-t^{2} / 2 \sigma_{p}^{2}} e^{i k M_{12} t} \\
& =A \delta\left[k_{0}-k / C(s)\right] e^{-\left(k M_{12} \sigma_{p}\right)^{2} / 2}
\end{aligned}
$$

with $\delta(\cdot)$ denoting the Dirac function.

As for the second term on the RHS of Eq. (12) we have

$$
\begin{aligned}
I_{2} & =-\left.\left.\frac{1}{2 \pi} \int d^{2} \boldsymbol{x}_{s} e^{-i k z_{s}} \int_{s_{0}}^{s} d s^{\prime} F\left(\tilde{\rho}_{1} ; z_{s^{\prime}} ; s^{\prime}\right)\right|_{z_{s^{\prime}}=z_{s^{\prime}}\left(\boldsymbol{x}_{s}\right)} \frac{\partial f_{0}\left(\boldsymbol{x}_{s^{\prime}} ; s^{\prime}\right)}{\partial p_{s^{\prime}}}\right|_{\boldsymbol{x}_{s^{\prime}}=\boldsymbol{x}_{s^{\prime}}\left(\boldsymbol{x}_{s}\right)} \\
& =-\frac{1}{2 \pi} \int d^{2} \boldsymbol{x}_{s^{\prime}} e^{-i k z_{s}\left(\boldsymbol{x}_{s^{\prime}}\right)} \int_{s_{0}}^{s} d s^{\prime} F\left(\tilde{\rho}_{1} ; z_{s^{\prime}} ; s^{\prime}\right) \frac{\partial f_{0}\left(\boldsymbol{x}_{s^{\prime}} ; s^{\prime}\right)}{\partial p_{s^{\prime}}} \\
& =-\frac{i k}{2 \pi} \int_{s_{0}}^{s} d s^{\prime} M_{12}\left(s^{\prime} \rightarrow s\right) \int d^{2} \boldsymbol{x}_{s^{\prime}} F\left(\tilde{\rho}_{1} ; z_{s^{\prime}} ; s^{\prime}\right) f_{0}\left(\boldsymbol{x}_{s^{\prime}} ; s^{\prime}\right) e^{-i k z_{s}\left(\boldsymbol{x}_{s^{\prime}}\right)} .
\end{aligned}
$$

The second equality above follows from a change of variables, the third from an integration by parts and $z_{s}\left(\boldsymbol{x}_{s^{\prime}}\right)=$ $\boldsymbol{M}_{11}\left(s^{\prime} \rightarrow s\right) z_{s^{\prime}}+\boldsymbol{M}_{12}\left(s^{\prime} \rightarrow s\right) p_{s^{\prime}}$. A further change of variables $\boldsymbol{x}_{s^{\prime}} \rightarrow \boldsymbol{x}_{0}$ yields

$$
I_{2}=-\frac{i k}{2 \pi} \int_{s_{0}}^{s} d s^{\prime} M_{12}\left(s^{\prime} \rightarrow s\right) \int d^{2} \boldsymbol{x}_{0} f_{0}\left(\boldsymbol{x}_{0} ; s_{0}\right) F\left[\tilde{\rho}_{1} ; z_{s^{\prime}}\left(\boldsymbol{x}_{0}\right) ; s^{\prime}\right] e^{-i k z_{s}\left[\boldsymbol{x}_{s^{\prime}}\left(\boldsymbol{x}_{0}\right)\right]}
$$

where we have made use of $f_{0}\left(\boldsymbol{x}_{s^{\prime}} ; s^{\prime}\right)=f_{0}\left(\boldsymbol{x}_{0} ; s_{0}\right)$. Observe that in the argument of the exponential function of the above expression we have $z_{s}\left[\boldsymbol{x}_{s^{\prime}}\left(\boldsymbol{x}_{0}\right)\right]=\left[M\left(s_{0} \rightarrow s\right) \boldsymbol{x}_{0}\right]_{1}=M_{11} z_{0}+M_{12} p_{0}$. 
Using the expression (9) for the collective force we find

$$
\begin{aligned}
I_{2}= & 4 \pi i \frac{I_{0}}{I_{A} Z_{0}} \frac{k}{2 \pi} \int_{-\infty}^{\infty} d k^{\prime} \int_{s_{0}}^{s} d s^{\prime} M_{12}\left(s^{\prime} \rightarrow s\right) Z\left(k^{\prime}, s^{\prime}\right) \\
& \times \tilde{\rho}_{1}\left(k^{\prime}, s^{\prime}\right) \int_{-\infty}^{\infty} d z_{0} \int_{-\infty}^{\infty} d p_{0} f_{0}\left(\boldsymbol{x}_{0} ; s_{0}\right) \\
& \times e^{i k^{\prime} z_{s^{\prime}}\left(\boldsymbol{x}_{0}\right)-i k z_{s}\left(\boldsymbol{x}_{0}\right)} .
\end{aligned}
$$

Again, we introduce the change of variable $p_{0} \rightarrow p_{0}=$ $t+h z_{0}$ and observe that

$$
\begin{aligned}
& k^{\prime} z_{s^{\prime}}\left(\boldsymbol{x}_{0}\right)-\left.k z_{s}\left(\boldsymbol{x}_{0}\right)\right|_{p_{0} \rightarrow p_{0}=t+h z_{0}} \\
& \quad=\left(\frac{k^{\prime}}{C\left(s^{\prime}\right)}-\frac{k}{C(s)}\right) z_{0}+\left(\frac{k^{\prime}}{M_{12}\left(s^{\prime}\right)}-\frac{k}{M_{12}(s)}\right) t .
\end{aligned}
$$

The integral on $z_{0}$ in (19) yields a Dirac function, allowing for a straightforward integration on $k^{\prime}$. After integration on $t$, we obtain

$$
\begin{aligned}
I_{2}= & 4 \pi i \frac{I_{0} k}{I_{A} Z_{0}} \int_{s_{0}}^{s} d s^{\prime} C\left(s^{\prime}\right) M_{12}\left(s^{\prime} \rightarrow s\right) Z\left(k^{\prime}, s^{\prime}\right) \\
& \times\left.\tilde{\rho}_{1}\left(k^{\prime} ; s^{\prime}\right) e^{-\left[k^{\prime} M_{12}\left(s^{\prime}\right)-k M_{12}(s)\right]^{2} \sigma_{p}^{2} / 2}\right|_{k^{\prime}=k C\left(s^{\prime}\right) / C(s)} .
\end{aligned}
$$

Finally, upon combining the various expressions evaluated so far, we arrive at the following integral equation for the Fourier components of the first-order density perturbation:

$$
\begin{aligned}
\tilde{\rho}_{1}(k ; s)= & e^{-\left[C(s) k_{0} M_{12} \sigma_{p}\right]^{2} / 2} A \delta\left[k_{0}-k / C(s)\right] \\
& +\int_{s_{0}}^{s} d s^{\prime} \hat{K}\left(s^{\prime}, s\right) \tilde{\rho}_{1}\left(k^{\prime} ; s^{\prime}\right)
\end{aligned}
$$

with kernel

$$
\begin{aligned}
\hat{K}\left(s^{\prime}, s\right)= & 4 \pi i \frac{I\left(s^{\prime}\right)}{I_{A}} k M_{12}\left(s^{\prime} \rightarrow s\right) \frac{Z\left(k^{\prime}, s^{\prime}\right)}{Z_{0}} \\
& \times\left. e^{-\left[k^{\prime} M_{12}\left(s^{\prime}\right)-k M_{12}(s)\right]^{2} \sigma_{p}^{2} / 2}\right|_{k^{\prime}=k C\left(s^{\prime}\right) / C(s),}
\end{aligned}
$$

where $I\left(s^{\prime}\right)=I_{0} C\left(s^{\prime}\right)$ is the beam current at $s^{\prime}$. We look for solutions of (22) in the space of generalized functions (distributions). Such solutions will have the form

$$
\begin{aligned}
\tilde{\rho}_{1}(k ; s) & =b(k ; s) \delta\left(k_{0}-\frac{k}{C(s)}\right) \\
& =b(k ; s) C(s) \delta\left[C(s) k_{0}-k\right],
\end{aligned}
$$

with the ordinary functions $b$ obeying the equation

$$
\begin{aligned}
b\left[C(s) k_{0} ; s\right]= & A e^{-\left[C(s) k_{0} M_{12} \sigma_{p}\right]^{2} / 2} \\
& +\int_{s_{0}}^{s} d s^{\prime} K\left(s^{\prime}, s\right) b\left[C\left(s^{\prime}\right) k_{0} ; s^{\prime}\right],
\end{aligned}
$$

with kernel $K\left(s^{\prime}, s\right)$ obtained from (23) with the substitution $k^{\prime} \rightarrow k_{0} C\left(s^{\prime}\right)$.

The integral equation (25) is our main result. Somewhat unexpectedly, but not too surprisingly, (25) turns out to be formally identical to the equation describing bunching in magnetic compressors $[5,6]$. We define the linear gain as the ratio of the amplitude of the perturbation at the exit $s=$ $s_{f}$ to that at the entrance $\left(s=s_{0}\right)$ :

$$
g\left(k_{0} ; s_{f}\right)=\frac{\left|b\left[C(s) k_{0} ; s_{f}\right]\right|}{|A|} .
$$

To summarize the meaning of the calculation carried out in this section: in linear approximation a sinusoidal perturbation with wave number $k_{0}$ to a beam charge density at the entrance of an rf compressor will maintain its sinusoidal form while the wave number evolves according to $k=$ $C(s) k_{0}$. The quantity $b(k ; s)$ or "bunching function," representing the amplitude of the sinusoidal perturbation relative to the local beam density $\rho(s)=C(s) \rho\left(s_{0}\right)$, is determined by solving the integral equation (25), where $A=b\left(k_{0} ; s_{0}\right)$ is the perturbation initial amplitude.

Collective effects in an rf compressor are largely dominated by space charge. We model these effects by means of an effective impedance $[9,11]$ relating the longitudinal component of the electric field and longitudinal chargedensity fluctuations in the frequency domain. In our model we neglect the possible dependence of the longitudinal component of the electric field on the radial coordinate and adopt a $1 \mathrm{D}$ expression for the space charge impedance of the form [12]

$$
Z(k, s)=\left.\frac{i Z_{0}}{\pi \gamma r_{b}} \frac{1-2 I_{1}(\xi) K_{1}(\xi)}{\xi}\right|_{\xi=k r_{b} / \gamma},
$$

where $Z_{0} \simeq 120 \pi$ is the vacuum impedance, and $I_{1}$ and $K_{1}$ are the modified Bessel functions of the first and the second kind.

Expression (27) is obtained [12] from transverse averaging of the longitudinal component of the electric filed of an infinitely long beam with circular cross section of radius $r_{b}$ (and uniform transverse density) perturbed by a small longitudinal modulation of wave number $k$. In principle, the radial dependence of the longitudinal electric field could be accounted for in the present framework but at the cost of increasing the dimensionality [8] of the integral

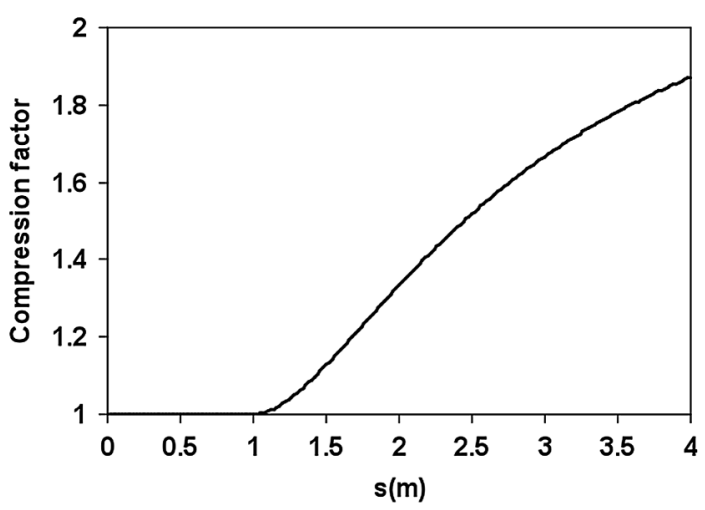

FIG. 3. Compression factor along the beam line. The value at exit is 1.87 . 

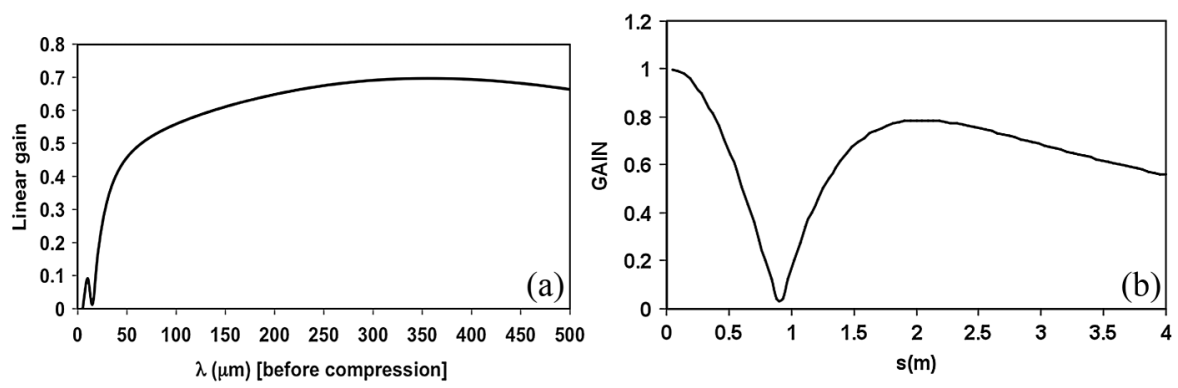

FIG. 4. (a) Linear gain at the exit of the rf compressor. (b) Linear gain as a function of the longitudinal position for a selected $(\lambda=100 \mu \mathrm{m})$ modulation wavelength.

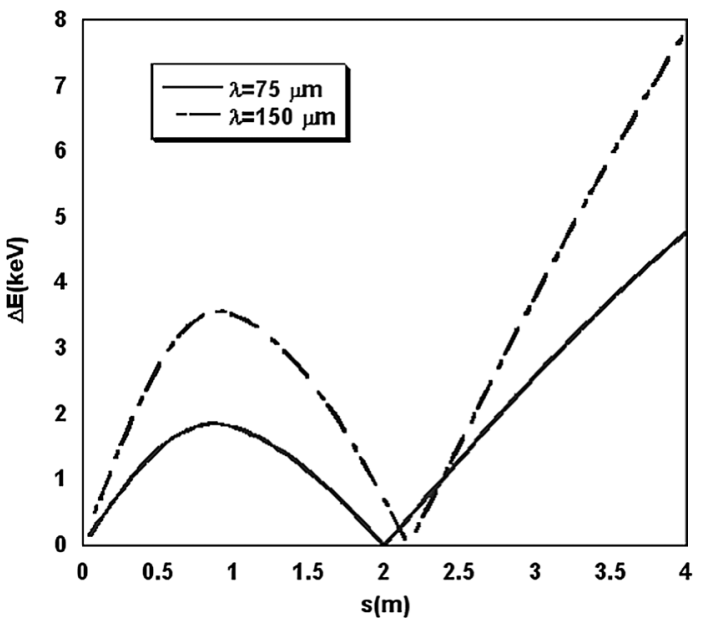

FIG. 5. Amplitude of the energy modulation amplitude along the rf compressor resulting from an initial density modulation with $10 \%$ amplitude relative to an initial current $I_{0}=100 \mathrm{~A}$. Other beam and rf compressor parameters are as in Figs. 3 and 4.

equation (25). Notice that in (27) the dependence on the longitudinal coordinate $s$ is through the parameter $r_{b}=$ $r_{b}(s)$, assumed to be known.

In Figs. 3 and 4 we show a numerical example for a model of beam line consisting of a $1 \mathrm{~m}$ drift followed by a $3 \mathrm{~m} \mathrm{rf}$ compressor. The beam is injected at $5.6 \mathrm{MeV}$ energy with $I_{0}=100 \mathrm{~A}$ peak current and vanishing uncorrelated energy spread. We assume a beam transversely uniform with circular cross section with radius $r_{b}=0.5 \mathrm{~mm}$ remaining constant through the beam line. The compression factor at the exit of the rf structure is 1.87. The gain curve as a function of the wavelength of the initial perturbation is reported in Fig. 4, and shows a maximum value of about 0.7 in the $\lambda \simeq 300 \mu \mathrm{m}$ region. The gain is $<1$ in the whole range of modulation wavelengths considered. Gains larger than unity could be observed but only in the presence of substantially higher current or compression factor.
Because of space charge an initial modulation in the charge density will induce an energy modulation along the beam. We are interested in determining to first order the amplitude of this latter modulation. To this end we introduce a new set of dynamical variables to remove the (in general nonvanishing) correlation energy/position

$$
\tilde{z}=z \quad \tilde{p}=p-h_{s} z
$$

and express the distribution function $f\left(x_{0} ; s\right)$ in terms of these new variables. In the above equation $h_{s}$ is the energy/ position correlation at $s$, which is related to the initial correlation $h_{0}$ at $s_{0}$ by $h_{s}=\left(M_{21}+h_{0} M_{22}\right) /\left(M_{11}+\right.$ $\left.h_{0} M_{12}\right)$.

Following the assumptions made in the previous section, we consider a beam distribution function $f=f_{0}+f_{1}$ in the form of the sum of a uniform zero-order density $f_{0}$ and a first-order perturbation $f_{1}$. The amplitude of the energy modulation is obtained by taking the FT of the energy centroid along the beam $\langle\tilde{p}\rangle=$ $\int f(\tilde{z}, \tilde{p} ; s) \tilde{p} d \tilde{p} / \int f(\tilde{z}, \tilde{p} ; s) d \tilde{p}$, a function of $\tilde{z}$ :

$$
\begin{aligned}
\Delta \tilde{p} & =\frac{1}{2 \pi} \int d \tilde{z} e^{-i k \tilde{z}}\langle\tilde{p}\rangle \\
& \simeq \frac{1}{2 \pi} \int d \tilde{z} e^{-i k \tilde{z}} \frac{\int f_{1}(\tilde{z}, \tilde{p} ; s) \tilde{p} d \tilde{p}}{\int f_{0}(\tilde{z}, \tilde{p} ; s) d \tilde{p}},
\end{aligned}
$$

where the last equation follows from first-order approximation and $\int_{-\infty}^{\infty} f_{0}(\tilde{z}, \tilde{p} ; s) \tilde{p} d \tilde{p}=0$ [see Eq. (7)]. The normalization factor $\int f_{0}(\tilde{z}, \tilde{p} ; s) d \tilde{p}$ is just the compression factor $C(s)$. To calculate $\int f_{1}(\tilde{z}, \tilde{p} ; s) \tilde{p} d \tilde{p}$ we make use of the first-order perturbation equation (11) for $f_{1}$, yielding

$$
\begin{aligned}
\Delta \tilde{p}= & -\frac{1}{2 \pi C(s)} \int \tilde{p}_{s} d \tilde{p}_{s} \int d \tilde{z}_{s} e^{-i k \tilde{z}_{s}} \int_{s_{0}}^{s} d s^{\prime} F\left(\tilde{\rho}_{1} ; z_{s^{\prime}} ; s^{\prime}\right) \\
& \times \frac{\partial f_{0}\left(\boldsymbol{x}_{s^{\prime}} ; s^{\prime}\right)}{\partial p_{s^{\prime}}} .
\end{aligned}
$$

After some algebra, here omitted for brevity, we find

$$
\Delta \tilde{p}=\left.\frac{4 \pi I_{0}}{I_{A} Z_{0}} \delta\left[k-k_{0} C(s)\right] C(s) \int_{s_{0}}^{s} d s^{\prime} b\left(k^{\prime} ; s^{\prime}\right) Z\left(k^{\prime}, s^{\prime}\right) e^{-\left[k^{\prime} M_{12}\left(s^{\prime} \rightarrow s\right) \sigma_{p}\right]^{2}}\left\{1-\left[k^{\prime} M_{12}\left(s^{\prime} \rightarrow s\right) \sigma_{p}\right]^{2}\right\}\right|_{k^{\prime}=k_{0} C\left(s^{\prime}\right)},
$$


with the bunching factor $b\left[k_{0} C\left(s^{\prime}\right) ; s^{\prime}\right]$ determined by solving Eq. (25).

In Fig. 5 we show two examples of energy modulations resulting from sinusoidal density perturbations with initial relative amplitude $A=0.1$ and wavelengths $\lambda=75$ and $150 \mu \mathrm{m}$.

\section{VALIDATION AGAINST MACROPARTICLE SIMULATIONS}

As a way to validate the model presented in the previous section, we carried out macroparticle simulations using the code TSTEP [13], a derivative of PARMELA [14]. We considered the evolution of a $1 \mathrm{nC}$ idealized flattop beam spanning a $10 \mathrm{deg}$ rf phase at $2856 \mathrm{MHz}$ (corresponding to about $3 \mathrm{~mm}$ and $I_{0}=100$ A peak current) and a range of initial charge-density sinusoidal perturbations with wavelength $\lambda$ between 50 and $300 \mu \mathrm{m}$. The upper limit of this range is determined by the need to consider wavelengths small enough compared to the bunch length so that the coasting beam approximation assumed in the analytical model may apply. The smallest modulation wavelength is limited by the statistical noise associated with use of a relatively small number of macroparticles. In the simulations we used up to $4.5 \times 10^{6}$ macroparticles, resulting in an acceptable compromise between numerical accuracy and computation time. The amplitude of the initial sinusoidal perturbation was set to $A=10 \%$.

The electron beam has vanishing initial energy-phase correlation and uncorrelated energy spread [i.e. $h_{0}=0$ and $\sigma_{p}=0$, see Eq. (7)].

We considered the $3 \mathrm{~m}$ long rf structure mentioned at the end of Sec. II preceded by a $0.6 \mathrm{~m}$ long drift. The presence of the drift is a realistic feature of any physical setup as the rf structure for compression compressor requires a certain separation from the exit of the gun. However, in these simulations, the exact value of the drift length was chosen to correspond (in the range of wavelength we considered and adopted initial beam conditions) to roughly a quarter wavelength of longitudinal plasma oscillation as this choice tends to maximize the amplitude of the modulation amplitude at the exit of the compressor (having started with pure density perturbations, i.e., no initial energy modulation).

The linear gain is calculated as the ratio between the amplitudes of the charge-density perturbation at the exit of the compressor and entrance of the leading $0.6 \mathrm{~m}$ drift. Care was taken to limit the analysis of the numerical data to the core of the bunch to minimize edge effects. The space charge forces were calculated by solving the Poisson equation on a grid with a $5 \mathrm{~mm}$ longitudinal span and number of mesh cells varying between 1200 and 2400 along the longitudinal coordinate.

We start the simulations with a beam with transverse uniform density and circular cross section of initial radius $r_{b}=2 \sigma_{x}=2 \sigma_{y}=2 \mathrm{~mm}$ and a convergent envelope $d r_{b} / d s<0$. It turns out that the plasma oscillation wavelength has a fairly strong dependence on the beam transverse radius $r_{b}$ and is therefore affected by the exact value of the initial beam convergence. This is exemplified in Fig. 6 where we show how different choices of the initial transverse conditions for the beam envelope affect the subsequent evolution of the beam radius (picture to the left) and the location of the first minimum for the linear gain for an initial perturbation of wavelength $\lambda=100 \mu \mathrm{m}$ (figure to the right). The minimum in the linear gain corresponds to a quarter of plasma oscillation wavelength from the entrance of the drift, which is where the initial density modulation is converted into energy modulation. See evidence of this in Fig. 7. For the remaining simulations presented in this paper we adopted the initial conditions for the beam envelope corresponding to the solid line in the left picture of Fig. 6. For simplicity, in the simulations we did not include solenoidal focusing along the rf structure, which would be required for emittance compensation [2], and let the beam expand freely.

Compression is controlled by moving the linac rf phase away from the crest toward the zero crossing of the rf field.
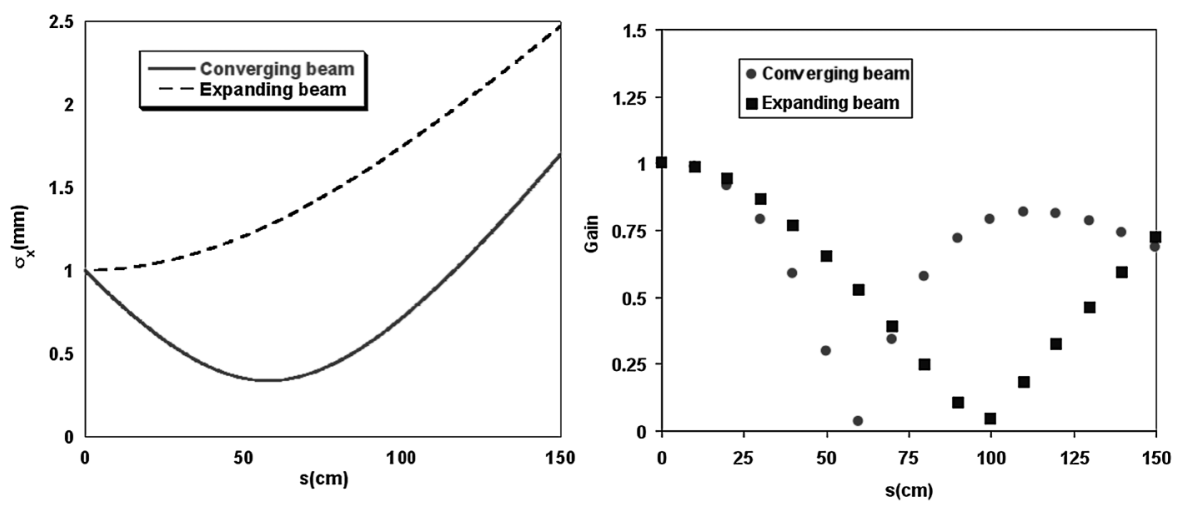

FIG. 6. Left: evolution of the transverse rms beam size $\sigma_{x}$ for a converging (solid line) and diverging (dashed line) beam. Right: corresponding linear gains for an initial modulation of wavelength $\lambda=100 \mu \mathrm{m}$. 

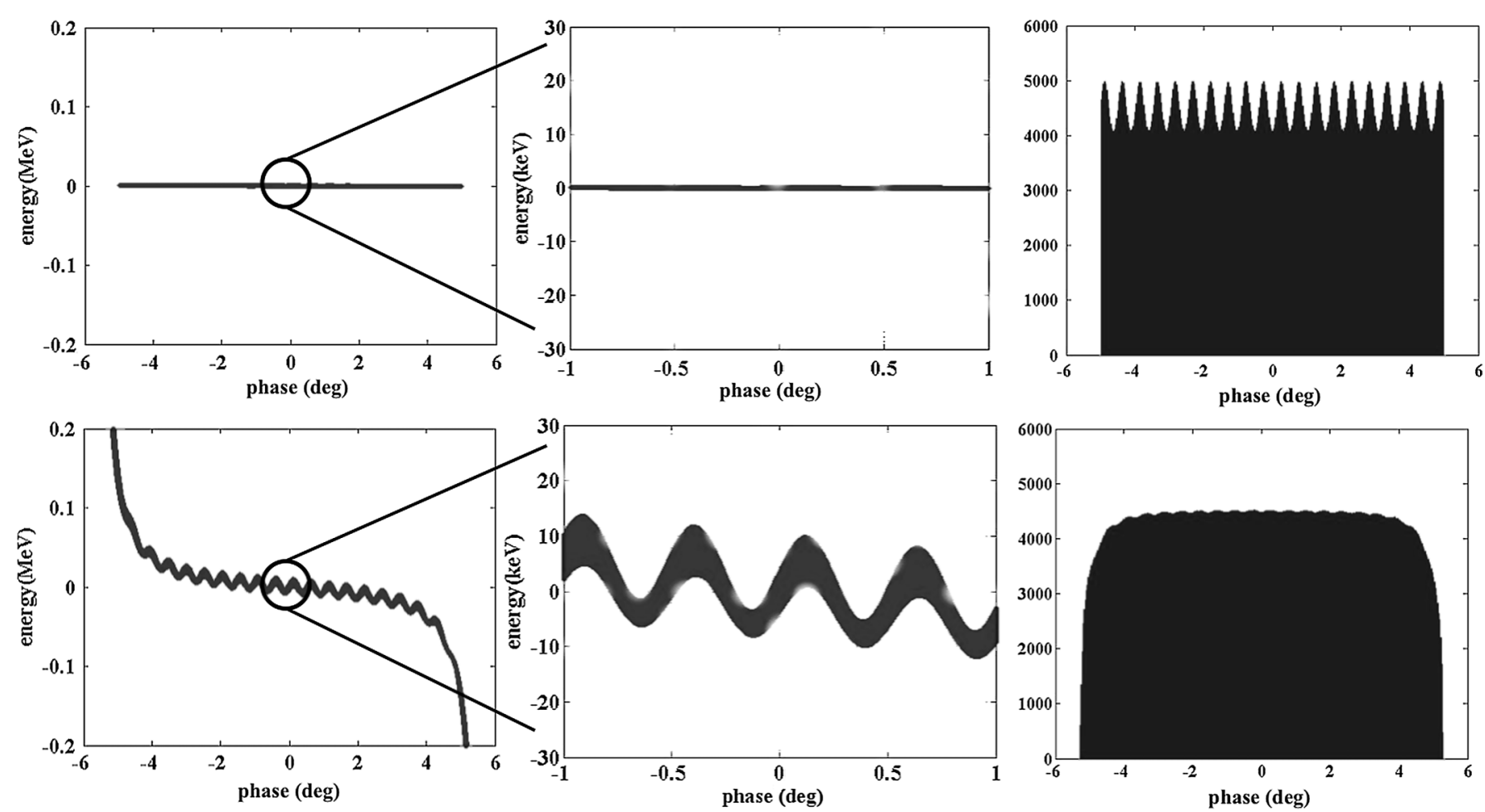

FIG. 7. Longitudinal phase space (left and center pictures) and bunch current distribution at the entrance of the leading drift (upper pictures) and entrance of the rf structure located $0.6 \mathrm{~m}$ downstream (lower pictures). The wavelength of the initial density modulation is $\lambda=150 \mu \mathrm{m}$. The longitudinal space coordinate is expressed in terms of rf phase with $10 \mathrm{deg}$ rf phase corresponding to about $3 \mathrm{~mm}$.

In this section we refer to the $\mathrm{rf}$ phase as defined as $\Psi(\operatorname{deg})=-90-\psi$, where $\psi$ is the phase defined in Sec. II.

To highlight the importance of a full account of space charge effects, we show a comparison between results obtained with two different settings where collective forces are turned on and off in the rf structure (while they are included through the leading drift in both cases), see Fig. 8 . The initial rf phase is $\Psi_{0}=-82 \mathrm{deg}$, corresponding to a compression factor $\sim 2$ ). It is seen that neglecting space charge in the rf structure (data points with round markers) leads to a gross overestimate of the gain.

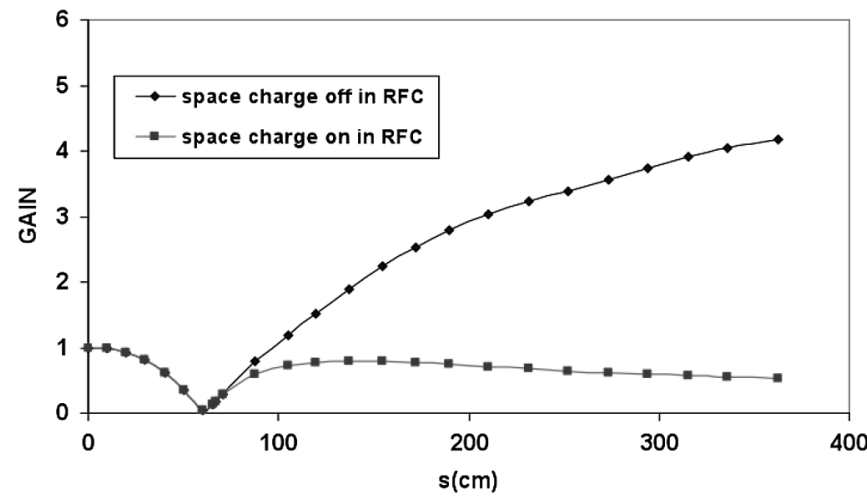

FIG. 8. Gain vs $z$ for $\operatorname{rf}$ phase $\Psi_{0}=-82 \operatorname{deg}(C \sim 2)$ with space charge on and off in the rf structure.
As the beam size variation affects in a sensitive way the results (see Fig. 6), it is important that when we make a comparison with the theory we account for the evolution of the beam radius along $s$. To this end, a high-order polynomial interpolation for the rms transverse size as a function of $s$ was carried out from data points extracted from the simulations. From analysis of the simulation data, it was found that the best agreement between the analytical model of Sec. III and simulations is obtained when in the expression for the impedance (27) we use the relationship $r_{b}=a \sigma_{x}$ with the factor $a$ defined as $a=1.95-0.001 \times$ $\lambda[\mu \mathrm{m}]$ instead of $a=2$, as would be expected for transversely uniform beams. The good agreement between simulations (solid line) and theory (dots), including the empirically adjusted factor $a$, is shown in Fig. 9. The good agreement also extends to the determination of the compression factor, Fig. 10 (with the compression factor from the simulation data calculated as the ratio $\sigma_{z 0} / \sigma_{z}$ between the initial $\sigma_{z 0}$ and final $\sigma_{z}$ rms bunch lengths).

Further simulation-vs-theory comparisons are reported in Figs. 11 and 12. In particular, in Fig. 11 we show the linear gain at the exit of the compressor over a range of perturbation wavelengths for a uncompressed $\left(\Psi_{0}=\right.$ $0 \mathrm{deg})$ and compressed $C=2\left(\Psi_{0}=-82 \mathrm{deg}\right)$ beam. Finally, in Fig. 13 we report the evolution of the amplitude of the energy modulation induced by an initial density modulation with $74 \mu \mathrm{m}$ wavelength. The energy modulation amplitude was retrieved from the simulation data by 

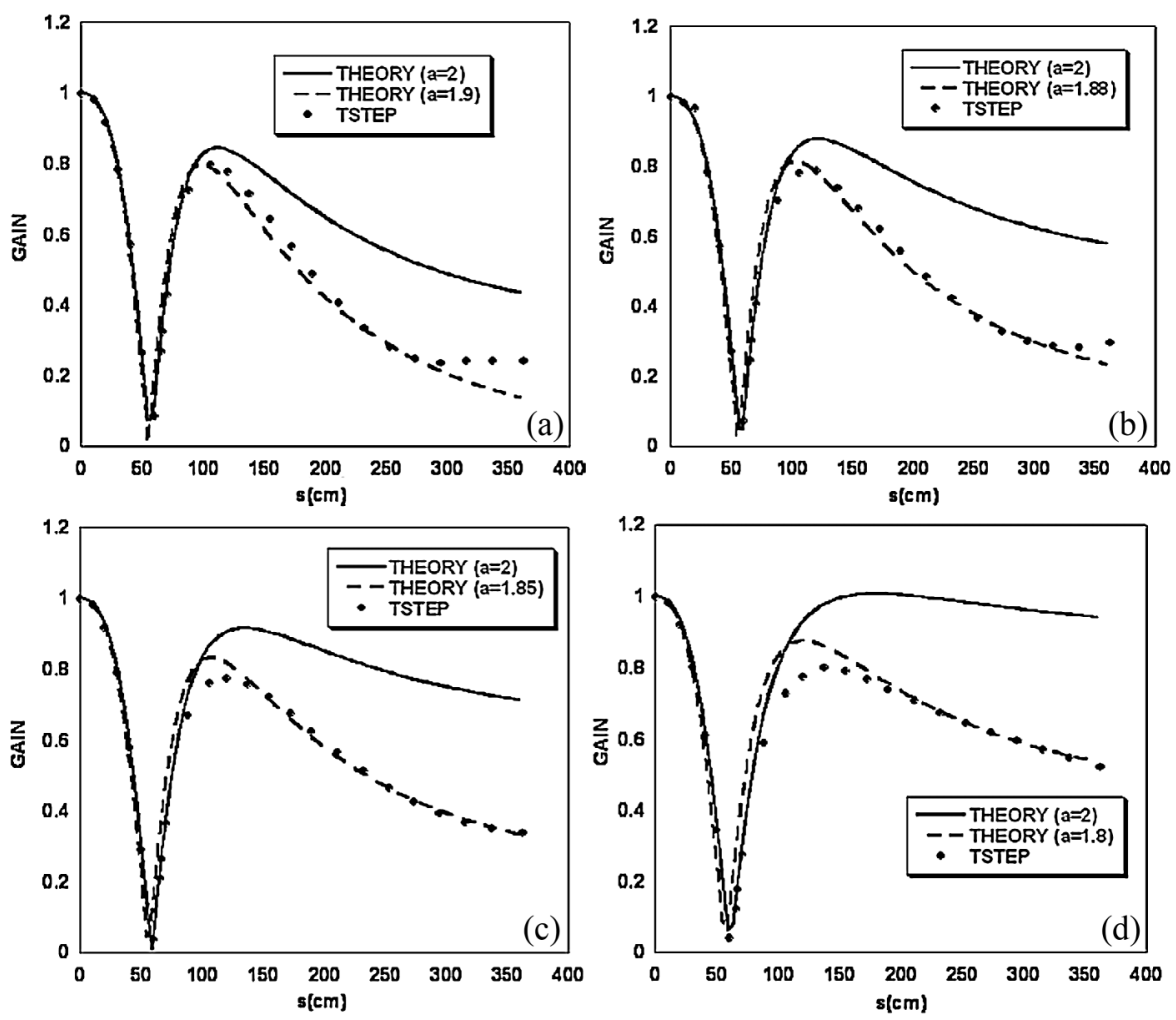

FIG. 9. Comparison between TSTEP simulations and linear theory for $\Psi_{0}=-82 \mathrm{deg}$ rf phase. The linear gain along the compressor is shown for four different initial modulation wavelengths: (a) $50 \mu \mathrm{m}$, (b) $75 \mu \mathrm{m}$, (c) $100 \mu \mathrm{m}$, and (d) $150 \mu \mathrm{m}$. The dashed line and solid lines were obtaining by setting $a=2$ and $a=1.95-0.001 \lambda[\mu \mathrm{m}]$, respectively, in the linear theory.

first removing the correlation phase energy in a window selected around the beam core in the longitudinal phase space and then carrying out a sinusoidal fit. An example of longitudinal beam phase space exhibiting the energy modulation is shown in Fig. 7. Notice that as it evolves the beam develops a finite energy spread due to the radial dependence of the space charge fields, which is missed by the 1D model of impedance adopted in our linear model.

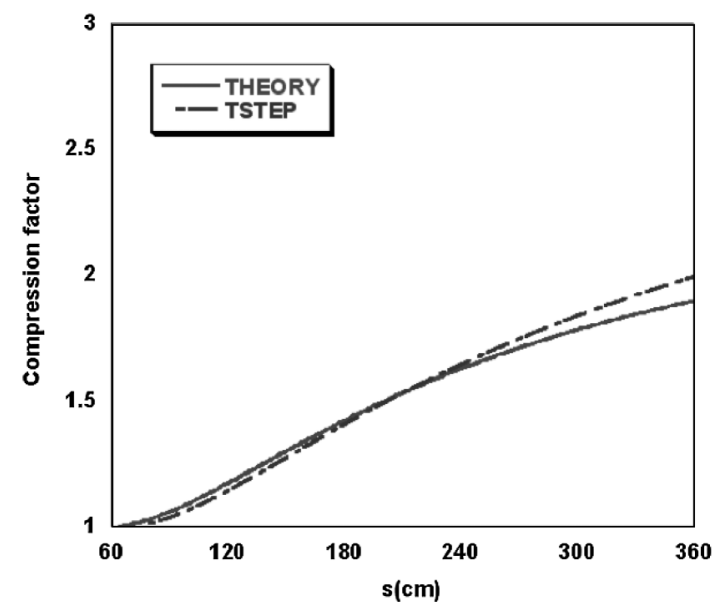

FIG. 10. Compression factor along the beam line (TSTEP simulations vs linear theory) for $\mathrm{rf}$ phase $\Psi_{0}=-82 \mathrm{deg}$.
Nonetheless, the linear model, including the empirical adjustment of the $a$ factor mentioned earlier, appears to reproduce quite well the amplitude of the energy modulation.

\section{CONCLUSIONS}

In this paper we have derived a linear theory for the amplitude gain of charge-density modulations of a beam passing through an rf compression system and have discussed a comparison against results from macroparticle simulations carried out with the code TSTEP. A satisfactory agreement with the linear theory is found when a parameter in the adopted 1D model for space charge effects is adjusted to account for an empirically determined dependence on the perturbation wavelength.

The numerical solutions of the linearized equations and the TSTEP simulations indicate that, for parameters of interest in typical FEL applications, the amplification of small initial density perturbations through an rf compressor tends to be quite modest (relative to the peak current) if not outright smaller than unity (i.e. implying damping of the initial perturbation) even in the absence of any uncorrelated energy-spread induced mixing. This result is not completely unexpected. We know, for example, that in a low-energy beam drifting in free space, and hence without compression, the amplitude of longitudinal plasma oscil- 

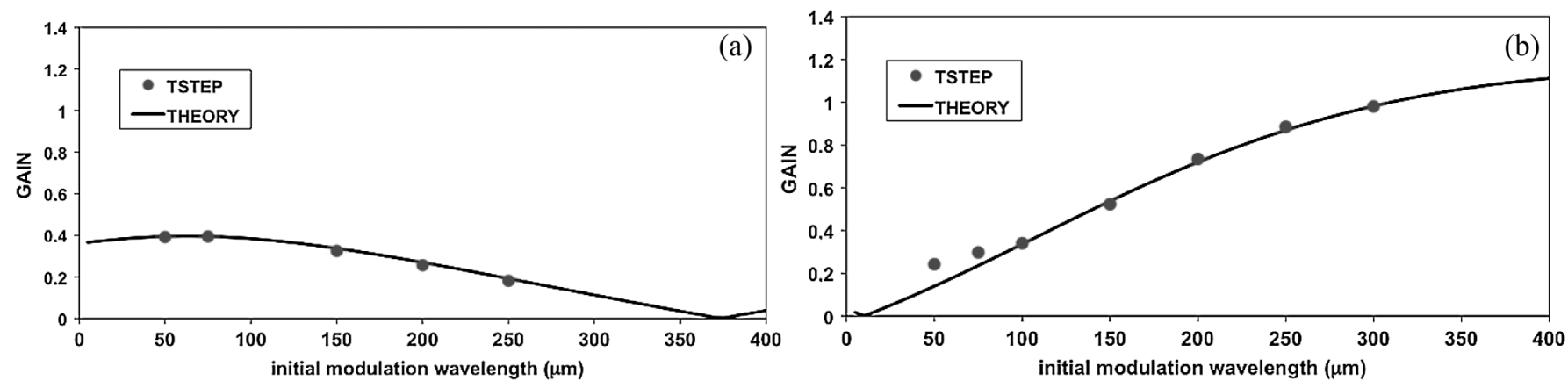

FIG. 11. Comparison between TSTEP simulations and linear theory for the linear gain through the bunch compressor over a spectrum of perturbation wavelengths; (a) is for a beam accelerated "on crest" (uncompressed), $C=1, \Psi_{0}=0$ deg, (b) is for a compression factor $C=2, \Psi_{0}=-82 \mathrm{deg}$.
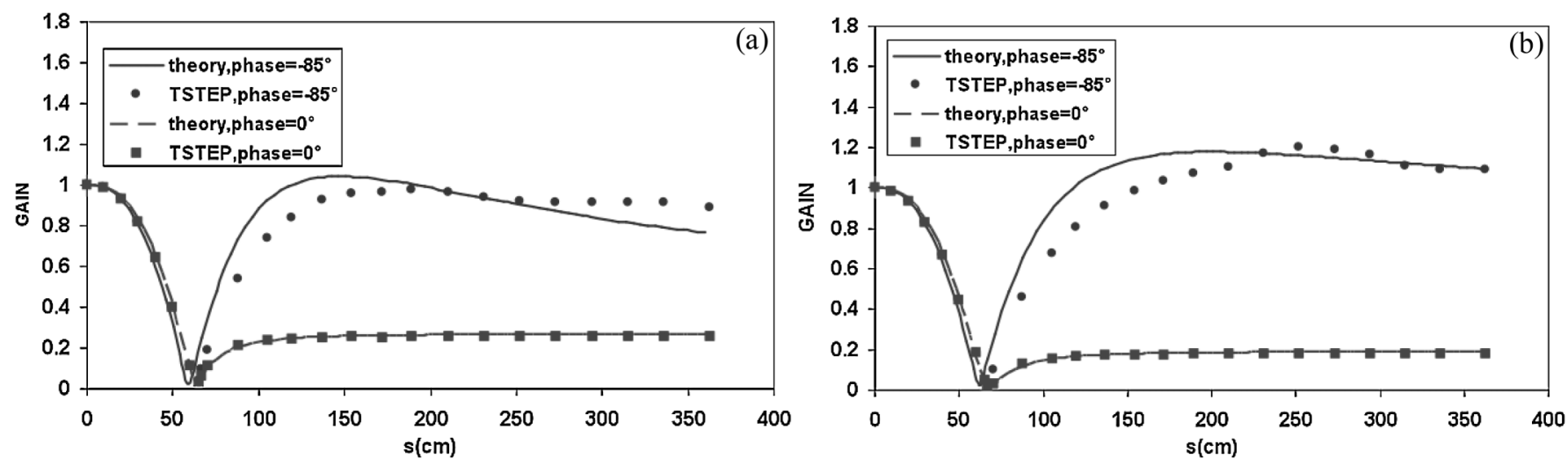

FIG. 12. Linear gain along the rf compressor for perturbation wavelengths (a) $\lambda=200 \mu \mathrm{m}$ and (b) $\lambda=250 \mu \mathrm{m}$ and two values of the initial rf phase.

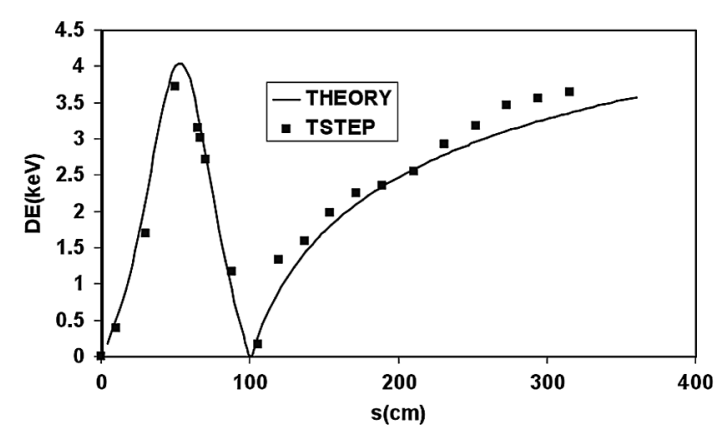

FIG. 13. Evolution of the amplitude of the energy modulation induced by an initial charge-density perturbation with wavelength $\lambda=74 \mu \mathrm{m}$. The prediction from linear theory (solid line) reproduces quite well the results from macroparticle simulations (dots).

lations remains constant. It turns out that, for moderate compression, velocity bunching changes the dynamics of longitudinal plasma oscillations from the case of freespace drifting only mildly - the main difference being in the adiabatic lengthening of the plasma oscillation wavelength and reduced strength of the space charge forces as the beam undergoes acceleration during compression. These results do not imply that the dynamics of smallamplitude density perturbations in the $\mathrm{rf}$ compressor should be neglected altogether as these perturbations can seed an instability downstream if further compression by magnetic chicanes is applied. Indeed, a scenario in which rf compression is supplemented by magnetic compression represents the most likely mode of operation envisioned for the accelerator drivers of FEL-based 4th generation light sources [3]. We expect that the theory elaborated in this paper will represent a useful tool in the evaluation of the compression schemes for these FEL sources. Finally, we should mention the possible relevance of our finding in connection with recent investigations of noise suppression in FEL injectors, see Gover et al. [15] and Nauser et al. [16].

\section{ACKNOWLEDGMENTS}

We acknowledge useful discussions with A. Zholents and J. Qiang. One of us, M. V., is grateful to the SPARC group for the hospitality during his stay at the INFN Laboratori Nazionali di Frascati. This work was supported in part by Department of Energy Contract No. DE-AC02$05 \mathrm{CH} 11231$. 
[1] L. Serafini and M. Ferrario, AIP Conf. Proc. 581, 87 (2001).

[2] M. Ferrario et al., Phys. Rev. Lett. 104, 054801 (2010).

[3] L. Palumbo et al., in Proceedings of the 11th European Particle Accelerator Conference, Genoa, 2008 (EPS-AG, Genoa, Italy, 2008), p. 121.

[4] E. L. Saldin et al., Nucl. Instrum. Methods Phys. Res., Sect. A 483, 516 (2002).

[5] S. Heifets, G. Stupakov, and S. Krinsky, Phys. Rev. ST Accel. Beams 5, 064401 (2002).

[6] Z. Huang and K.-J. Kim, Phys. Rev. ST Accel. Beams 5, 074401 (2002).

[7] M. Venturini, Phys. Rev. ST Accel. Beams 10, 104401 (2007).

[8] J. Wu, Z. Huang, and P. Emma, Phys. Rev. ST Accel. Beams 11, 040701 (2008).

[9] M. Venturini, Phys. Rev. ST Accel. Beams 11, 034401 (2008).
[10] D. Alesini et al., in Proceedings of the 11th European Particle Accelerator Conference, Genoa, 2008 (Ref. [3]), p. 2169.

[11] J. Rosenzweig, C. Pellegrini, L. Serafini, C. Ternieden, and G. Travish, Nucl. Instrum. Methods Phys. Res., Sect. A 393, 376 (1997).

[12] J. Qiang, R. D. Ryne, M. Venturini, A. A. Zholents, and I. V. Pogorelov, Phys. Rev. ST Accel. Beams 12, 100702 (2009).

[13] L. M. Young (private communication).

[14] L. M. Young, Los Alamos National Laboratory Report No. LA-UR-96-1835.

[15] A. Gover and E. Dyunin, Phys. Rev. Lett. 102, 154801 (2009)

[16] A. Nause, E. Dyunin, and A. Gover, J. Appl. Phys. 107, 103101 (2010). 